\title{
Uma Contribuição ao Desenvolvimento de Indicadores de Desempenho para Ativos Intangíveis Organizacionais
}

\author{
Claudelino Martins Dias Junior ${ }^{1}$ \\ Marcus Vinícius A. de Lima \\ Marcos Baptista Lopez Dalmau ${ }^{3}$
}

\section{Resumo}

O presente artigo cataloga modelos gerenciais que são utilizados e/ou adaptados em suas concepções originais e evidenciam a importância dos ativos intangíveis internos como efetivos elementos da sustentabilidade econômica de uma empresa. Assim, demonstra-se a metodologia de Teixeira (2005) de determinação de indicadores de flexibilidade para manufatura, a metodologia de França (2004) para o estabelecimento de indicadores para ativos intangíveis organizacionais e a metodologia de Hoss (2003) de avaliação quantitativa e qualitativa para os valores dos ativos intangíveis. Tal intento objetivou, considerando a participação dos valores dos ativos intangíveis, o desenvolvimento de indicadores de desempenho para estes mesmos ativos, no sentido de se obter melhores níveis de eficiência operacional na gestão de uma unidade de manufatura.

Palavras-chave: Indicadores de desempenho. Ativos Intangíveis. Manufatura e Objetivos Organizacionais.

\footnotetext{
${ }^{1}$ Professor do Departamento de Ciências da Administração da Universidade Federal de Santa Catarina - UFSC, Coordenador Acadêmico do PNAP. End.: Universidade Federal de Santa Catarina, Departamento de Ciências da Administração - Sala 124, Campus Reitor João David Ferreira Lima, Trindade, Florianópolis - SC. CEP: 88040-970 - Brasil. E-mail: marcus.lima@cse.ufsc.br . 2 Professor do Departamento de Ciências da Administração da Universidade Federal de Santa Catarina - UFSC, Coordenador Adjunto do CPGA. End.: Universidade Federal de Santa Catarina, Departamento de Ciências da Administração - Sala 124, Campus Reitor João David Ferreira Lima - Trindade - Florianópolis - SC. CEP: 88040-970 - Brasil. E-mail: marcus.lima@cse.ufsc.br.

3 Doutor em Engenharia de Produção pela Universidade Federal de Santa Catarina - UFSC. Professor do Departamento de Ciências da Administração da Universidade Federal de Santa Catarina - UFSC. End.: Universidade Federal de Santa Catarina, Departamento de Ciências da Administração - Sala 124, Campus Reitor João David Ferreira Lima - Trindade - Florianópolis - SC. CEP: 88040 970 - Brasil. E-mail: dalmau@cse.ufsc.br.

Artigo recebido em: 27/10/2010. Aceito em: 30/04/2011. Membro do Corpo Editorial Científico responsável pelo processo editorial: Emerson Antonio Maccari.
} 


\section{Introdução}

A tentativa das organizações construírem proposições de valor mais ajustadas às percepções de seus clientes e/ou consumidores, por vezes, encontra-se limitada pela utilização de instrumentos de avaliação e mensuração do valor dos ativos originários de uma economia sustentada pela visão de racionalização de recursos tangíveis e com perspectivas orientadas a quantificações de ganhos financeiros e, por conseguinte, não alicerçados por um efetivo reconhecimento da contribuição dos ativos intangíveis internos que dinamizam suas atividades de produção mais elementares.

Percebe-se que a necessidade do estabelecimento de indicadores evolutivos do desempenho dos processos de produção considerando a presença de ativos intangíveis internos, de forma a estabelecerem-se critérios de investimentos em ativos de mesma natureza que sejam considerados críticos para manutenção da atividade de manufatura (DIAS JUNIOR, 2007a).

Dessa forma, apresentam-se instrumentos metodológicos que evidenciam a contribuição dos ativos intangíveis no universo das organizações, tais como: a escolha de indicadores organizacionais mais ajustados à natureza dos ativos intangíveis, que possibilitem seus reconhecimentos como agentes de produção de riqueza; a quantificação dos níveis de contribuição desses ativos no ambiente de manufatura; a consequente forma de determinação da priorização dos ativos intangíveis como objetos de alocação de recursos.

\section{Referencial Teórico}

Como pressuposto fundamental para a concepção de uma metodologia que permeie a necessidade de reconhecimento dos ativos intangíveis, surge a necessidade de estabelecer-se uma coerente interdependência na valoração dos ativos intangíveis utilizados no contexto organizacional, no sentido de compatibilizar sua gestão com os resultados econômico-financeiros previamente estimados.

A utilização de indicadores precisos dependerá do tipo de produtos, da organização e da estratégia de desenvolvimento de produtos, tornando-se imprópria a ideia de recomendação de indicadores generalizáveis, o importante 
é que um quadro de indicadores estabelecidos pelas organizações contemple dimensões financeiras e não-financeiras, de resultados e de meios (SILVA, 2001).

Embora a maioria dos autores classifique de diferentes formas e determine o foco sob diferentes aspectos a utilização de indicadores de desempenho organizacional, Pandolfi (2005) indica a necessidade de que estes estejam alinhados com estratégias competitivas e objetivos estratégicos. Nesse sentido, apresentam-se alguns modelos gerenciais concebidos que perpassam tal preocupação.

\subsection{O Modelo de Teixeira na Determinação da Flexibilidade na Manufatura}

Tendo o foco inicial no planejamento estratégico e procurando ressaltar as mudanças fundamentais que deverão preencher os objetivos estratégicos, Teixeira (2005) procura medir a margem de contribuição do elemento intangível (flexibilidade) definindo prioridades de investimentos para este no ambiente de manufatura. Inicialmente, propõe, a partir de uma listagem de priorização de todos objetivos estratégicos gerais, a criação de indicadores coadunados à implantação do planejamento estratégico do negócio.

Da metodologia proposta por Teixeira (2005) para a determinação de indicadores de desempenho para diferentes tipologias de flexibilidades, é adaptada na efetiva proposição de desenvolvimento dos indicadores de desempenho relacionados aos ativos intangíveis internos e presentes no contexto da manufatura. Paralelamente, tal modelo, igualmente, auxilia a determinação de objetivos setoriais de manufatura no posicionamento de produtos tidos como estratégicos, que sejam assim priorizáveis, em função de sua importância para a sustentabilidade econômica da empresa. Ao passo que se busca uma compatibilização destes com os objetivos setoriais da unidade de manufatura e com a escolha de indicadores mais ajustados (Críticos) à gestão dos ativos intangíveis internos. 


\subsection{O Modelo de Determinação de Indicadores de Ativos Intangíveis de França}

França (2004) desenvolveu um modelo de indicadores para o monitoramento da presença de ativos intangíveis organizacionais, estabelecendo como principais pressupostos do estudo os seguintes:

a) a avaliação dos ativos intangíveis constitui um processo multidimensional que encerra a necessidade de reconhecimento, mapeamento, medição e valoração;

b) a avaliação formal dos ativos intangíveis requer contextualização na determinação de indicadores, estando estes sujeitos à diversidade de percepções sujeitas à imprecisão e a incerteza;

c) o processo de avaliação dos ativos intangíveis pode suportar o crescimento da empresa e fornecer alternativas de apoio dos processos de decisão, vinculando ações às estratégias de longo prazo, de forma a garantir a sobrevivência da empresa.

Assim, o reconhecimento e o mapeamento de indicadores da presença de ativos intangíveis se dariam a partir de uma percepção dos colaboradores organizacionais dos processos internos, como também de interlocutores externos à atividade (FRANÇA, 2004). Para escolha preliminar dos indicadores de ativos intangíveis utilizou-se as seguintes categorias de ativos intangíveis, sendo: relacionamento de negócios; estruturas internas, competências humanas, cidadania social, saúde ambiental e identidade corporativa.

Em conformidade com os objetivos propostos, França (2004) discorre sobre a importância da utilização do método como forma de reconhecer e mapear indicadores que estejam diretamente ligados à atividade de agregação de valor organizacional e que, consequentemente, possam apontar a presença de ativos intangíveis organizacionais.

\subsection{O Modelo de Avaliação de Ativos Intangíveis de Hoss}

Para Hoss (2003), a criação de valor representativa da presença de ativos intangíveis, assim como França (2004), estaria ligada à consideração de perspectivas internas e externas à organização, subdivididas em quatro quadrantes, 
sendo: três pertencentes à perspectiva interna (humano, processos e estrutural) e o quarto seria a perspectiva externa (ambiental).

Analisando essas perspectivas sob dois focos, sendo: presente-passado $e$ passado-futuro. Para o entendimento do foco presente-passado voltar-se-ia aos quadrantes pertencentes a processos e estrutura, posto que o elemento estrutura seria o primeiro na constituição da organização seguidos dos processos, para então, com o apoio do humano, conseguir-se ofertar produtos e serviços. $\mathrm{O}$ foco presente-futuro atenta para os quadrantes humano e ambiental, pois a prestação de serviço no presente, mesmo que dentro das condições de competitividade, não constitui a continuidade das operações.

Assim, Hoss (2003) procura demonstrar a relação de interdependência dos ativos intangíveis sob a forma de interatividade dos quadrantes expostos, formando o que denomina como Lucro Intangível Ajustado (LIA), conforme Figura 1.

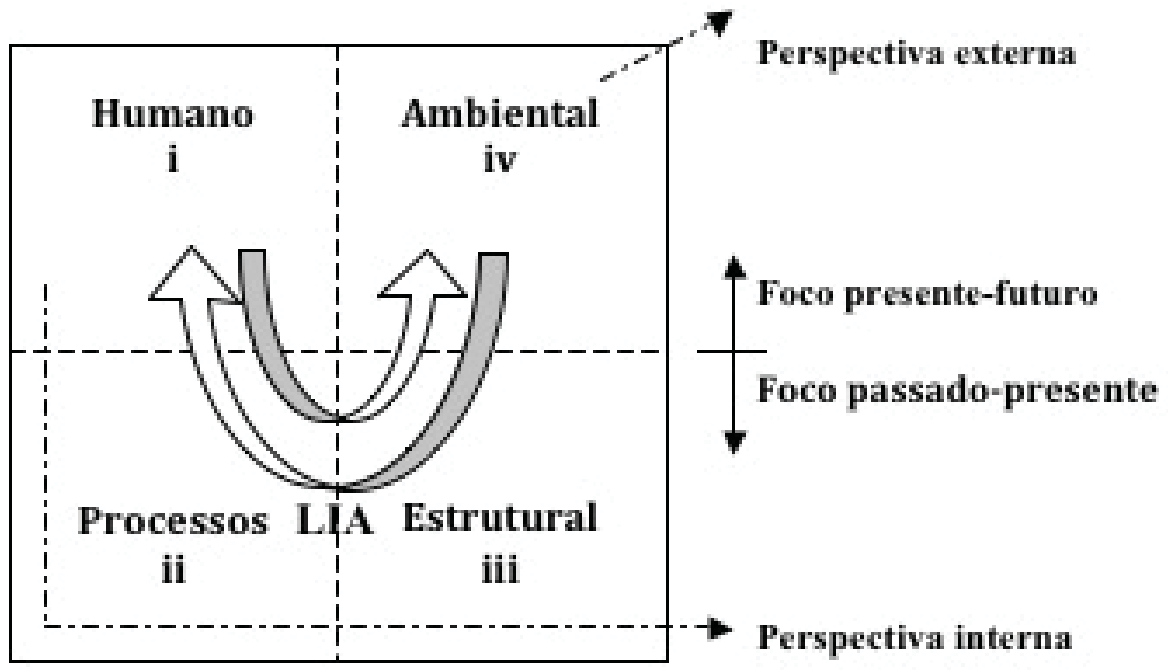

Figura 1: Mensuração dos Ativos Intangíveis em Perspectiva Fonte: Adaptada de Hoss (2003)

Na busca de uma avaliação qualitativa e quantitativa, Hoss (2003) sugere a eleição de variáveis que sejam utilizadas como guias no processo de avaliação do valor dos ativos intangíveis. 
De outra parte, as variáveis quantitativas seriam representadas pelo esforço na aplicação de riquezas para a maximização do negócio, considerando-se igualmente os quadrantes definidos para o cálculo das variáveis qualitativas (humano, processos, estrutural e ambiental). Com a definição dessas variáveis, passa-se ao cálculo do valor dos ativos intangíveis.

A seguir, descrevem-se as possibilidades de utilização das metodologias descritas no cálculo dos ativos intangíveis organizacionais e na determinação de indicadores de desempenho para a gestão desses mesmos ativos.

\section{Determinação de Indicadores de Desempenho para Ativos Intangíveis}

A metodologia de Hoss (2003) é utilizada no cálculo do valor dos ativos intangíveis internos identificados como próprios da organização, esse cálculo visa ter em conta as variáveis qualitativas (humanas, de processos, estruturais e ambientais), que representam os quadrantes necessários e considerados na determinação do valor dos ativos intangíveis.

Para a efetiva utilização de ativos tangíveis e intangíveis internos relacionados aos PEPs (Produtos Estratégicos Priorizáveis) visa-se determinar e hierarquizar objetivos de manufatura que possam determinar melhores níveis de eficiência operacional, ou seja, próximos a 1 (um) para PEPs com AIIs relacionados. Para a determinação desses objetivos propõe-se que tal questionamento possa ser respondido, sendo: "Quais outras variáveis internas estão envolvidas na racionalização da utilização de ativos tangíveis e ativos intangíveis internos para a produção dos PEPs?". Ao passo que, das variáveis consideradas necessárias à racionalização dos recursos tangíveis e intangíveis utilizados para produção de cada PEP, deva-se descrevê-las na forma de objetivo a serem perseguido, conforme descrito na segunda coluna do Quadro 1. 


\begin{tabular}{|c|c|c|c|}
\hline $\begin{array}{c}\text { Produtos } \\
\text { Estratégicos } \\
\text { Priorizados } \\
\text { (PEPs) }\end{array}$ & $\begin{array}{c}\text { Descrição dos Objetivos de } \\
\text { Manufatura (OMs) }\end{array}$ & $\begin{array}{c}\text { Nível de } \\
\text { Prioridade } \\
\text { dos OMs } \\
\text { (peso) }\end{array}$ & $\begin{array}{c}\text { Ordem de } \\
\text { Hierarqui- } \\
\text { zação dos } \\
\text { OMs }\end{array}$ \\
\hline \multirow{3}{*}{$\mathrm{PEP}_{4}$} & modernizar o parque fabril $\left(\mathrm{OM}_{1}\right)$ & 10 & $1^{\circ}$ \\
\cline { 2 - 4 } & melhorar os insumos de produção $\left(\mathrm{OM}_{5}\right)$ & 7 & $5^{\circ}$ \\
\cline { 2 - 4 } & diminuir tempos de entrega $\left(\mathrm{OM}_{2}\right)$ & 9 & $2^{\circ}$ \\
\hline \multirow{3}{*}{$\mathrm{PEP}_{3}$} & qualificar mão-de-obra $\left(\mathrm{OM}_{3}\right)$ & 8 & $3^{\circ}$ \\
\cline { 2 - 5 } & informatizar a produção $\left(\mathrm{OM}_{4}\right)$ & 7 & $4^{\circ}$ \\
\cline { 2 - 5 } & desenvolver parcerias $\left(\mathrm{OM}_{6}\right)$ & 6 & $6^{\circ}$ \\
\hline
\end{tabular}

Quadro 1: Exemplo de Determinação e Hierarquização dos Objetivos de Manufatura Fonte: Adaptado de Teixeira (2005)

Como forma de hierarquizar os objetivos descritos passa-se a uma ordem de priorização utilizando-se uma escala de pesos que varie de 1 a 10 . Posteriormente, procede-se ao cálculo das médias simples dos pesos obtidos por cada objetivo, segundo as percepções individuais dos envolvidos (gerentes de produção). A ordem de hierarquização dos objetivos de manufatura obedecerá à maior pontuação obtida em escala decrescente. Obtendo-se pontuações iguais, a hierarquização se dará pela relação do objetivo com ao setor de manufatura, no qual o PEP obtiver menor índice de eficiência.

Estabelecidos os objetivos de manufatura (OMs), por meio de consenso entre os gestores das unidades setoriais, quanto à pertinência da execução de cada OM, far-se-ão as seguintes indagações, respectivamente:

a) "este objetivo pode ser alcançado no contexto da manufatura?";

b) "se perseguido este objetivo no contexto da manufatura, qual setor se encarrega de sua execução?".

Dessa forma, o alcance dos objetivos de manufatura se dará por meio de ações setoriais, doravante denominados OSs (ver exemplo no Quadro 2). 


\begin{tabular}{|c|c|c|c|}
\hline PEPs & $\begin{array}{l}\text { Objetivos de Manufatura } \\
\text { (OMs) }\end{array}$ & $\begin{array}{l}\text { Setores da } \\
\text { Manufatura }\end{array}$ & $\begin{array}{c}\text { Objetivos Setoriais } \\
\text { da Manufatura }\end{array}$ \\
\hline \multirow{3}{*}{$\mathrm{PEP}_{4}$} & \multirow{3}{*}{$\begin{array}{l}\text { - modernizar o parque fabril (OM1); } \\
\text { - diminuir tempos de entregas } \\
\text { (OM2); } \\
\text { - melhorar os insumos de produção } \\
\text { (OM5). }\end{array}$} & Setor de PCP & $\left(\mathrm{OS}_{4}\right)$ \\
\hline & & Setor de Logística & \\
\hline & & Setor de Manutenção & $\left(\mathrm{OS}_{1}\right)$ \\
\hline \multirow{3}{*}{$\mathrm{PEP}_{3}$} & \multirow{3}{*}{$\begin{array}{l}\text { - qualificar mão-de-obra (OM3); } \\
\text { - informatizar a produção (OM4); } \\
\text { - desenvolver parcerias (OM6). }\end{array}$} & Setor de PCP & $\left(\mathrm{OS}_{6}\right)$ \\
\hline & & Setor de Logística & $\left(\mathrm{OS}_{2}\right)$ \\
\hline & & Setor de Manutenção & \\
\hline
\end{tabular}

Quadro 2: Exemplo de Estabelecimento dos Objetivos Setoriais da Manufatura Fonte: Adaptado de Teixeira (2005)

Para tanto, para se definir a relação de indicadores de desempenho da manufatura (IDs) com os objetivos setoriais na produção dos PEPs deve-se adotar como referência a seguinte pergunta: "Os indicadores de desempenho da manufatura possuem alguma relação com os Alls (Ativos Intangíveis Internos)?" (ver exemplo em Quadro 3).

\begin{tabular}{|c|c|c|c|c|}
\hline PEPs & $\begin{array}{l}\text { Objetivos } \\
\text { Setoriais da } \\
\text { Manufatura }\end{array}$ & $\begin{array}{l}\text { Indicadores de } \\
\text { Desempenho } \\
\text { da Manufatura } \\
\left(\text { ID }_{\text {pep }}\right)\end{array}$ & $\begin{array}{c}\text { Relação } \\
\text { do ID com } \\
\text { AIIs }\end{array}$ & $\begin{array}{c}\text { Indicador de } \\
\text { Desempenho } \\
\text { para AIIs - } \\
\text { IDAIIs }\end{array}$ \\
\hline \multirow{5}{*}{$\mathrm{PEP}_{4}$} & \multirow{3}{*}{$\begin{array}{c}\left(\mathrm{OS}_{1}\right)-\text { modernizar } \\
\text { o parque fabril }\end{array}$} & $\mathrm{ID}_{(\mathrm{pep} 4) \mathrm{a}}$ & SIM & IDAII $_{(\mathrm{pep} 4) \mathrm{a}}$ \\
\hline & & $\mathrm{ID}_{(\mathrm{pep} 4) \mathrm{b}}$ & SIM & IDAII $_{(\text {pep } 4) b}$ \\
\hline & & $\mathrm{ID}_{(\mathrm{pep} 4) \mathrm{c}}$ & NÃO & - \\
\hline & \multirow{2}{*}{$\begin{array}{l}\left(\mathrm{OS}_{2}\right)-\text { diminuir } \\
\text { tempos de entregas }\end{array}$} & $\mathrm{ID}_{(\mathrm{pep} 4) \mathrm{d}}$ & SIM & IDAII $_{(\mathrm{pep} 4) \mathrm{c}}$ \\
\hline & & $\mathrm{ID}_{(\mathrm{pep} 4) e}$ & $\mathrm{NA} O$ & - \\
\hline \multirow{3}{*}{$\mathrm{PEP}_{3}$} & \multirow{2}{*}{$\begin{array}{l}\left(\mathrm{OS}_{4}\right) \text { - informati- } \\
\text { zar a produção }\end{array}$} & $\mathrm{ID}_{(\mathrm{pep} 3) \mathrm{a}}$ & SIM & IDAII $_{(\mathrm{pep} 3) \mathrm{a}}$ \\
\hline & & $\mathrm{ID}_{(\mathrm{pep} 3) \mathrm{b}}$ & SIM & IDAII $_{(\mathrm{pep} 3) \mathrm{b}}$ \\
\hline & $\begin{array}{c}\left(\mathrm{OS}_{6}\right) \text { - desenvol- } \\
\text { ver parcerias }\end{array}$ & $\mathrm{ID}_{(\mathrm{pep} 3) \mathrm{c}}$ & SIM & IDAII $_{(\mathrm{pep} 3) \mathrm{c}}$ \\
\hline
\end{tabular}

Quadro 3: Exemplo de Identificação dos Indicadores de Desempenho Relacionados a AIIs IDAIIs

Fonte: Adaptado de Teixeira (2005) 
Criadas as condições para o estabelecimento dos indicadores que orientem a atividade de manufatura, relacionados aos Alls e tendentes a melhoria dos níveis de eficiência para a produção dos PEPs, esses indicadores passam a denominar-se Indicadores de Desempenho de Ativos Intangíveis Internos (IDAIIs).

Para determinar o grau de importância de cada IDAII, a escala de gradação a ser adotada será uma adaptação do modelo proposto por Teixeira (2005), conforme descrito na Quadro 4.

\begin{tabular}{|c|c|}
\hline Nível de Influência & Pesos \\
\hline Fortíssima & 9 \\
\hline Muita & 6 \\
\hline Razoável & 3 \\
\hline Pouca & 1 \\
\hline Nenhuma & - \\
\hline
\end{tabular}

Quadro 4: Pesos para Graus de Importância Fonte: Adaptado de Teixeira (2005)

O procedimento a ser adotado para identificação do grau de importância de cada IDAII consistirá na indagação: "Qual o grau de importância dos AIIs envolvidos para o alcance do objetivo setorial da manufatura?". Paralelamente, para se determinar o grau de importância do ID a ser adotado por cada setor, far-se-á a seguinte pergunta: "Qual o grau de importância do IDAII para o setor envolvido?" (ver Tabela 1). 
Tabela 1: Exemplo de Matriz de Relações entre Objetivos Setoriais de Manufatura e os Indicadores de Desempenho de Ativos Intangíveis Internos

\begin{tabular}{|c|c|c|c|c|c|c|c|}
\hline \multirow[b]{2}{*}{$\begin{array}{l}\text { Obje- } \\
\text { tivos } \\
\text { Seto- } \\
\text { riais } \\
\text { da } \\
\text { Manu- } \\
\text { fatura }\end{array}$} & \multirow[b]{2}{*}{$\begin{array}{c}\text { Grau } \\
\text { de Im- } \\
\text { portân- } \\
\text { cia dos } \\
\text { AIIs } \\
\text { (peso) }\end{array}$} & \multirow{2}{*}{$\begin{array}{l}\text { Indicador } \\
\text { de De- } \\
\text { sempenho } \\
\text { de Ativos } \\
\text { Intan- } \\
\text { gíveis } \\
\text { Internos } \\
\text { IDAIIs }\end{array}$} & \multicolumn{3}{|c|}{ Setores de Manufatura } & \multirow[b]{2}{*}{$\begin{array}{c}\text { Peso } \\
\text { Rela- } \\
\text { tivo } \Sigma \\
\text { IDAII }_{(\text {pep) }}\end{array}$} & \multirow[b]{2}{*}{$\begin{array}{c}\text { Nível de } \\
\text { Importância } \\
\text { do IDAII em } \\
\text { Relação aos } \\
\text { Setores da } \\
\text { Manufatura }\end{array}$} \\
\hline & & & 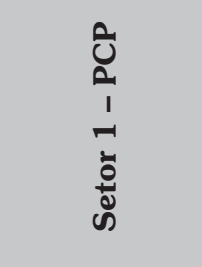 &  & ڤ & & \\
\hline \multirow{4}{*}{$\mathrm{OS}_{1}$} & \multirow{2}{*}{9} & \multirow{2}{*}{ IDAII $_{(\text {pep4)a }}$} & & & 9 & \multirow{2}{*}{81} & \multirow{2}{*}{$\begin{array}{l}(81 \times 100) / 288 \\
\quad=28,13 \%\end{array}$} \\
\hline & & & & & 81 & & \\
\hline & \multirow{2}{*}{9} & \multirow{2}{*}{ IDAII $_{(\text {pep4)b }}$} & & & 9 & \multirow{2}{*}{81} & \multirow{2}{*}{$28,13 \%$} \\
\hline & & & & & 81 & & \\
\hline \multirow{2}{*}{$\mathrm{OS}_{2}$} & \multirow{2}{*}{6} & \multirow{2}{*}{ IDAII $_{(\mathrm{pep} 4) \mathrm{d}}$} & & 9 & & \multirow{2}{*}{54} & \multirow{2}{*}{$18,75 \%$} \\
\hline & & & & 54 & & & \\
\hline \multirow{4}{*}{$\mathrm{OS}_{4}$} & \multirow{2}{*}{6} & \multirow{2}{*}{ IDAII $_{(\text {pep3)a }}$} & 6 & & & \multirow{2}{*}{36} & \multirow{2}{*}{$12,50 \%$} \\
\hline & & & 36 & & & & \\
\hline & \multirow{2}{*}{1} & \multirow{2}{*}{ IDAII $_{(\text {pep3)b }}$} & 9 & & & \multirow{2}{*}{9} & \multirow{2}{*}{$3,12 \%$} \\
\hline & & & 9 & & & & \\
\hline \multirow{2}{*}{$\mathrm{OS}_{6}$} & \multirow{2}{*}{3} & \multirow{2}{*}{ IDAII $_{(\text {pep3)c }}$} & 9 & & & \multirow{2}{*}{27} & \multirow{2}{*}{$9,37 \%$} \\
\hline & & & 27 & & & & \\
\hline \multicolumn{3}{|c|}{ Peso Relativo ( $\sum$ Setor) } & $\begin{array}{l}36+9+ \\
26=72\end{array}$ & 54 & $\begin{array}{c}81+81= \\
162\end{array}$ & 288 & - \\
\hline \multicolumn{3}{|c|}{$\begin{array}{l}\text { Nível de Importância do Se- } \\
\text { tor para atingir os OMs }\end{array}$} & $\begin{array}{c}(72 \times 100) / \\
288=25,00 \%\end{array}$ & $18,75 \%$ & $56,25 \%$ & - & $100 \%$ \\
\hline
\end{tabular}

Fonte: Adaptada de Teixeira (2005)

De acordo com o observado em Tabela 1 pode-se definir o nível de envolvimento de cada setor como os IDAIIs. Na Tabela 2 exemplificam-se os cálculos dos graus de importância dos AIIs em relação a cada IDAII. 
Tabela 2: Exemplo de Matriz do Grau de Influência dos AIls

\begin{tabular}{|c|c|c|c|c|c|c|c|c|c|c|c|c|c|c|}
\hline \multicolumn{3}{|c|}{$\begin{array}{c}\text { Setores da } \\
\text { Manufatura }\end{array}$} & \multicolumn{11}{|c|}{ Setor 1 - PCP } & ... \\
\hline \multirow{3}{*}{\multicolumn{3}{|c|}{$\begin{array}{c}\text { Níveis de } \\
\text { Contribuição }\end{array}$}} & \multicolumn{11}{|c|}{ Indicadores de Desempenho de Ativos Intangíveis Internos - IDAIIs } & \\
\hline & & & \multirow{2}{*}{\multicolumn{2}{|c|}{$\begin{array}{c}\text { IDAII }_{(\text {pep4)a }} \\
0,2813 \\
\end{array}$}} & \multirow{2}{*}{\multicolumn{2}{|c|}{$\begin{array}{c}\text { IDAII }_{(\text {pep4)b }} \\
0,2813\end{array}$}} & \multirow{2}{*}{\multicolumn{2}{|c|}{\begin{tabular}{|c|} 
IDAII $_{\text {(pep4)d }}$ \\
0,1875 \\
\end{tabular}}} & \multirow{2}{*}{\multicolumn{2}{|c|}{$\frac{\text { IDAII }_{(\mathrm{pep} 3) \mathrm{a}}}{0,1250}$}} & \multirow{2}{*}{\multicolumn{2}{|c|}{$\frac{\text { IDAII }_{(\mathbf{p e p} 3) \mathrm{b}}}{0,0312}$}} & IDAII $_{(\text {pep } 3) \mathrm{c}}$ & $\cdots$ \\
\hline & & & & & & & & & & & & & 0,0937 & ... \\
\hline \multirow{4}{*}{$\stackrel{0}{Z}$} & \multirow{2}{*}{\multicolumn{2}{|c|}{$\begin{array}{c}\text { Contratos de } \\
\text { Relaciona- } \\
\text { mento com } \\
\text { Clientes }\end{array}$}} & \multicolumn{2}{|c|}{9} & \multicolumn{2}{|c|}{1} & \multicolumn{2}{|c|}{3} & \multicolumn{2}{|c|}{1} & \multicolumn{2}{|c|}{6} & 1 & $\ldots$ \\
\hline & & & \multicolumn{2}{|c|}{2,5317} & \multicolumn{2}{|c|}{0,2813} & \multicolumn{2}{|c|}{0,5625} & \multicolumn{2}{|c|}{0,1250} & \multicolumn{2}{|c|}{0,1278} & 0,0937 & $\ldots$ \\
\hline & \multirow{2}{*}{\multicolumn{2}{|c|}{$\begin{array}{c}\text { Contratos de } \\
\text { Franquia }\end{array}$}} & \multicolumn{2}{|c|}{6} & \multicolumn{2}{|c|}{6} & & & \multicolumn{2}{|c|}{1} & \multicolumn{2}{|c|}{3} & 3 & $\ldots$ \\
\hline & & & \multicolumn{2}{|c|}{1,6878} & \multicolumn{2}{|c|}{1,6878} & & & \multicolumn{2}{|c|}{0,1250} & \multicolumn{2}{|c|}{0,0936} & 0,2811 & $\ldots$ \\
\hline \multicolumn{3}{|c|}{$\Sigma$ Pesos Relativos } & & 195 & & & & & & & & 214 & 0,3748 & \\
\hline & aus de Ir & luên- & & $24 \%$ & & $15 \%$ & & & & & & $5 \%$ & $3,13 \%$ & $\ldots$ \\
\hline $\begin{array}{l}\text { cia } \\
\text { cac } \\
\text { Se } \\
\end{array}$ & $\begin{array}{l}\text { dos AIIs } \\
\text { la Indica } \\
\text { tor - GI } \\
\end{array}$ & $\begin{array}{l}\text { sobre } \\
\text { or do } \\
\text { I (\%) } \\
\end{array}$ & & & & & & AII $\Sigma$ & 63 , & & & & & $\ldots$ \\
\hline & & & & Setor & -1 & anute & ção & & & & & $\begin{array}{c}\text { Setor } \\
\mathbf{n}\end{array}$ & $\Sigma$ & $\begin{array}{l}\text { GIAII } \\
\text { PEPs }\end{array}$ \\
\hline & ndicad & es de & Deser & penhe & de $A$ & ivos I & tang & eis In & erno & - IDA & & $\cdots$ & & $(\%)$ \\
\hline $\mathrm{ID}$ & $\mathrm{AII}_{(\text {pep4)a }}$ & IDAIl & pep 4$) \mathrm{b}$ & IDAII & & IDAI & ep3)a & IDAI & & IDA & & $\ldots$ & & \\
\hline & 2813 & $0,2 \varepsilon$ & & $0,1 \varepsilon$ & & 0,1 & & 0,0 & & 0,0 & & $\ldots$ & & \\
\hline & 3 & & & 3 & &  & & - & & & & $\ldots$ & 67529 & $5640 \%$ \\
\hline & 8439 & & & 0,5 & & 0,7 & & 0,0 & & & & $\ldots$ & 0,1529 & \\
\hline & & 3 & & & & 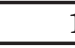 & & 3 & & & & $\ldots$ & 52189 & $4360 \%$ \\
\hline & & 0,8 & & & & 0,1 & & 0,0 & & 0,2 & & $\ldots$ & & \\
\hline & 8439 & 0,8 & & 0,5 & & 0,8 & & 0,1 & & & & & 11,9718 & \\
\hline & $05 \%$ & 7,0 & & 4,7 & & 7,3 & & 1,0 & & & & & & \\
\hline & & & & $\mathbf{G I}$ & II $\Sigma$ & 36,5 & & & & & & $\ldots$ & - & $100 \%$ \\
\hline
\end{tabular}

Fonte: Adaptada de Teixeira (2005)

Observa-se que o cálculo da importância relativa de cada indicador não foi efetuado no exemplo de Tabela 2 para todos os setores da manufatura considerados. No entanto, estão explicitados os graus de importância relativos a cada AII apontado.

Com o objetivo de utilizar os AIls como instrumentos efetivos de aumento da eficiência operacional da manufatura, faz-se necessário efetuar os cálculos 
de suas margens de contribuição e graus de criticidade para o alcance dos objetivos setoriais (OSs). Para tanto, serão considerados apenas IDAIls que encerrem um grau de contribuição superior a 0,1 (ver exemplo em Tabela 2), posto que os IDAIIs com graus de contribuição < 0,1 encerram contribuições marginais não relevantes na determinação do grau de criticidade dos Alls (ver exemplo em Tabela 3).

Tabela 3: Exemplo de Cálculo do Grau de Criticidade dos AIIs

\begin{tabular}{|c|c|c|c|c|c|}
\hline \multirow[b]{2}{*}{$\begin{array}{c}\text { Tipos de } \\
\text { AIIs }\end{array}$} & \multirow[b]{2}{*}{$\begin{array}{c}\text { Grau de } \\
\text { Influên- } \\
\text { cia Total } \\
\text { do AII }\end{array}$} & \multicolumn{2}{|c|}{ Setor 1 - PCP } & \multicolumn{2}{|c|}{ Setor 2 - Manutenção } \\
\hline & & $\begin{array}{c}\text { IDAIIs Ativos }\left(\mathrm{n}^{\circ}\right. \\
\text { total) }\end{array}$ & $\begin{array}{c}\text { Grau de } \\
\text { Criti- } \\
\text { cidade } \\
\text { do AII - } \\
\text { GCAII }_{(\mathrm{S} 1)}\end{array}$ & $\begin{array}{c}\text { IDAIIs Ativos ( } \mathbf{n}^{\circ} \\
\text { total) }\end{array}$ & $\begin{array}{c}\text { Grau de } \\
\text { Criti- } \\
\text { cidade } \\
\text { do AII - } \\
\text { GCAII }_{(\mathrm{S} 2)}\end{array}$ \\
\hline $\begin{array}{c}\text { Contratos de } \\
\text { Relaciona- } \\
\text { mento com } \\
\text { Clientes }\end{array}$ & $\begin{array}{c}56,40 \%= \\
0,5640\end{array}$ & $\begin{array}{c}(2,5317+0,2813+0,5625 \\
\quad+0,1250+0,1278) / 5\end{array}$ & $\begin{array}{l}\text { GCAIIIP }_{(\mathrm{S} 1)} \\
=0,4092\end{array}$ & $\begin{array}{c}(0,8439+0,5625+ \\
0,7500+0,8433) / 4\end{array}$ & $\begin{array}{l}\text { GCAIIP }_{(\mathrm{S} 2)} \\
=0,4229\end{array}$ \\
\hline $\begin{array}{l}\text { Contratos } \\
\text { de Franquia }\end{array}$ & $\begin{array}{c}43,60 \%= \\
0,4360\end{array}$ & $\begin{array}{c}(1,6878+1,6878+0,1250 \\
+0,2811) / 4\end{array}$ & $\begin{array}{l}\text { GCAIIIP }_{(\mathrm{S} 1)} \\
=0,4122\end{array}$ & $\begin{array}{c}(0,8439+0,1250+ \\
0,2811) / 3\end{array}$ & $\begin{array}{l}\text { GCAIIP }_{(\mathrm{S} 2)} \\
=0,1816\end{array}$ \\
\hline
\end{tabular}

Fonte: Adaptada de Teixeira (2005)

Para o cálculo do grau de criticidade setorial dos Alls procede-se à multiplicação do grau de influência total do AII pelo resultado da média dos IDAIIs ativos $(>0,1)$. Pode-se observar que, dado o resultado obtido, em exemplo de Tabela 3, para o setor de PCP o AII (Contratos de Franquia) apresenta-se como o mais crítico, de forma inversa para o setor de manutenção.

Para definir a ordem de prioridade para os Alls utilizar-se-á uma escala de pesos, com a seguinte gradação: mais crítico (peso 9); crítico (peso 6) ou menos crítico (peso 3). Ressalta-se que as faixas de enquadramento ficam a cargo da percepção comum dos componentes da equipe condutora do modelo. O Quadro 5 mostra a ordem de prioridade dos AIIs, por meio da representação de seus graus de contribuição em cada setor analisado, servindo de base para visualização inicial da importância de cada no contexto em análise. 


\begin{tabular}{|c|c|c|c|c|c|}
\hline AIIs & $\begin{array}{c}\text { Ordem de } \\
\text { Prioridade }\end{array}$ & $\begin{array}{c}\text { GCAII } \\
(\mathbf{S n})\end{array}$ & AII(Sn) & $\begin{array}{c}\text { Peso da } \\
\text { Situação do } \\
\text { Arítico }\end{array}$ & $\begin{array}{c}\text { Situação } \\
\text { do AII } \\
\text { Crítico }\end{array}$ \\
\hline $\begin{array}{c}\text { Contratos de Relacio- } \\
\text { namento com Clientes }\end{array}$ & $1^{\circ}$ & 0,4229 & $(\mathrm{~S} 2)$ & Mais Crítico & 9 \\
\hline Contratos de Franquia & $2^{\circ}$ & 0,4122 & $(\mathrm{~S} 1)$ & Crítico & 6 \\
\hline $\begin{array}{c}\text { Contratos de Relacio- } \\
\text { namento com Clientes }\end{array}$ & $3^{\circ}$ & 0,4092 & $(\mathrm{~S} 1)$ & Crítico & 6 \\
\hline Contratos de Franquia & $4^{\circ}$ & 0,1816 & $(\mathrm{~S} 2)$ & Menos Crítico & 3 \\
\hline
\end{tabular}

Quadro 5: Exemplo da Ordem de Prioridade dos AIIs Críticos Fonte: Adaptado de Teixeira (2005)

Considerando que o AII (Contratos de Relacionamento com Clientes) exerce maior influência no Setor 1 (PCP) e no Setor 2 (Manutenção) configura igualmente como crítico, conclui-se que tal AII determina o bom desempenho de ambos os setores, sendo crítico para o alcance de melhores níveis de eficiência.

Objetiva-se a alocação de recursos em Alls considerados críticos ao aumento dos níveis de eficiência no contexto da manufatura de forma que esses Alls proporcionem a utilização mais racional de outras categorias de ativos, sejam esses tangíveis e intangíveis.

De forma a identificar os elementos que forneçam condições à criação $e$ à manutenção dos AIls, considera-se que esses possam ser representados por: recursos humanos; processos; estrutura organizacional e fatores ambientais. Para cada um deles é definido um peso em relação ao AII crítico, multiplicando-se pelo GCAII (Grau de Criticidade do Ativo Intangível Interno) em cada setor e pelo peso do respectivo AII (ver exemplo em Quadro 5). Obter-se-á, assim, os pesos relativos de cada AIl em cada setor analisado, bem como a representação percentual de cada elemento formador do mesmo ativo, conforme descrito nos exemplos de Tabela 4 e Tabela 5. 
Tabela 4: Exemplo de Matriz dos Elementos dos AIIs - Setor 1

\begin{tabular}{|c|c|c|c|c|c|c|c|c|}
\hline \multirow[b]{2}{*}{ 臬 } & \multirow[b]{2}{*}{ AlI Crítico } & \multirow[b]{2}{*}{$\begin{array}{c}\text { GCAII/ } \\
\text { S1 }\end{array}$} & \multirow[b]{2}{*}{$\begin{array}{c}\text { Peso } \\
\text { do AII } \\
\text { Crítico }\end{array}$} & \multicolumn{4}{|c|}{ Elementos dos AIIs } & \multirow[b]{2}{*}{$\begin{array}{l}\text { ¿Pesos } \\
\text { Relati- } \\
\text { vos (\%) }\end{array}$} \\
\hline & & & & 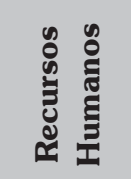 & $\begin{array}{l}0 \\
0 \\
8 \\
8 \\
0 \\
0 \\
0\end{array}$ &  &  & \\
\hline \multirow{5}{*}{ 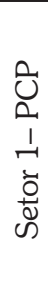 } & \multirow{2}{*}{$\begin{array}{c}\text { Contratos de } \\
\text { Relacionamento } \\
\text { com Clientes }\end{array}$} & \multirow[b]{2}{*}{0,4092} & \multirow[b]{2}{*}{6} & 9 & 6 & 6 & 9 & \multirow{2}{*}{$\begin{array}{c}73,6568 \\
(65,05 \%)\end{array}$} \\
\hline & & & & 22,0968 & 14,7312 & 14,7312 & 22,0968 & \\
\hline & \multirow{2}{*}{$\begin{array}{c}\text { Contratos de } \\
\text { Franquia }\end{array}$} & \multirow{2}{*}{0,4122} & \multirow{2}{*}{6} & 6 & 6 & 1 & 3 & \multirow{2}{*}{$\begin{array}{c}39,5712 \\
(34,95 \%)\end{array}$} \\
\hline & & & & 14,8392 & 14,8392 & 2,4732 & 7,4196 & \\
\hline & TOTAL (\%) & - & - & $\begin{array}{c}36,936 \\
(32,62 \%)\end{array}$ & $\begin{array}{l}29,5704 \\
(26,12 \%)\end{array}$ & $\begin{array}{l}17,2044 \\
(15,19 \%)\end{array}$ & $\begin{array}{l}29,5164 \\
(26,07 \%)\end{array}$ & $\begin{array}{c}113,2272 \\
(100,00 \%)\end{array}$ \\
\hline
\end{tabular}

Fonte: Adaptada de Teixeira (2005)

Tabela 5: Exemplo de Matriz dos Elementos dos AIIs - Setor 2

\begin{tabular}{|c|c|c|c|c|c|c|c|c|}
\hline \multirow[b]{2}{*}{$\frac{\mathscr{c}}{0}$} & \multirow[b]{2}{*}{ AlI Crítico } & \multirow[b]{2}{*}{$\begin{array}{c}\text { GCAII/ } \\
\text { S1 }\end{array}$} & \multirow[b]{2}{*}{$\begin{array}{c}\text { Peso } \\
\text { do AII } \\
\text { Crítico }\end{array}$} & \multicolumn{4}{|c|}{ Elementos dos AIIs } & \multirow[b]{2}{*}{$\begin{array}{l}\sum \text { Pesos } \\
\text { Relati- } \\
\text { vos (\%) }\end{array}$} \\
\hline & & & & 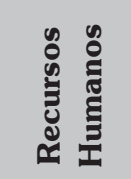 &  & 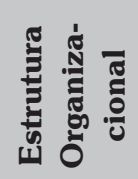 & 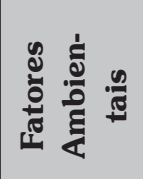 & \\
\hline \multirow{5}{*}{  } & \multirow{2}{*}{$\begin{array}{l}\text { Contratos de } \\
\text { Relacionamento } \\
\text { com Clientes }\end{array}$} & \multirow{2}{*}{0,4229} & \multirow{2}{*}{9} & 6 & 6 & 3 & 3 & \multirow{2}{*}{$\begin{array}{c}68,5098 \\
(85,69 \%)\end{array}$} \\
\hline & & & & 22,8366 & 22,8366 & 11,4183 & 11,4183 & \\
\hline & \multirow{2}{*}{$\begin{array}{c}\text { Contratos de } \\
\text { Franquia }\end{array}$} & \multirow{2}{*}{0,1816} & \multirow{2}{*}{3} & 6 & 6 & 6 & 3 & \multirow{2}{*}{$\begin{array}{c}11,4408 \\
(14,31 \%)\end{array}$} \\
\hline & & & & 3,2688 & 3,2688 & 3,2688 & 1,6344 & \\
\hline & TOTAL (\%) & - & - & $\begin{array}{c}26,1054 \\
(32,65 \%)\end{array}$ & $\begin{array}{c}26,1054 \\
(32,65 \%)\end{array}$ & $\begin{array}{c}14,6871 \\
(18,37 \%)\end{array}$ & \begin{tabular}{|c|}
13,0527 \\
$(16,33 \%)$
\end{tabular} & $\begin{array}{c}79,9506 \\
(100,00 \%)\end{array}$ \\
\hline
\end{tabular}

Fonte: Adaptada de Teixeira (2005)

Procede-se agora ao cálculo das margens de contribuição dos elementos dos AIIs, por meio da seguinte fórmula:

\section{MC $=($ Peso Relativo do Elemento AII $)$ x $100 / \sum$ Pesos Relativos}

Obtendo-se tais índices como segue:

a) para o elemento Recursos Humanos, AII Crítico (Contratos de Relacionamentos com Clientes), Setor 1:

$M C=22,0968 \times 100 / 113,2272=19,51 \%$; 
b) para o elemento Fatores Ambientais, AII Crítico (Contratos de Franquia), Setor 2:

$\mathrm{MC}=1,6344 \times 100 / 79,9506=2,04 \%$;

c) para o elemento Estrutura Organizacional, AII Crítico (Contratos de Relacionamentos com Clientes), Setor 1:

$\mathrm{MC}=14,7312 \times 100 / 113,2272=13,01 \%$.

A Tabela 6 e Tabela 7 demonstram tais cálculos.

Tabela 6: Exemplo de Matriz de Margem de Contribuição dos Elementos dos AIIs - Setor 1

\begin{tabular}{|c|c|c|c|c|c|c|}
\hline \multirow[b]{2}{*}{ 兵 } & \multirow[b]{2}{*}{ AII Crítico } & \multicolumn{4}{|c|}{ ELEMENTOS DOS AIIs } & \multirow{2}{*}{$\begin{array}{c}\text { ¿Mar- } \\
\text { gem de } \\
\text { Contri- } \\
\text { buição } \\
(\%)\end{array}$} \\
\hline & & $\begin{array}{l}\text { Recursos } \\
\text { Humanos }\end{array}$ & Processos & $\begin{array}{c}\text { Estrutura } \\
\text { Organiza- } \\
\text { cional }\end{array}$ & $\begin{array}{c}\text { Fatores } \\
\text { Ambien- } \\
\text { tais }\end{array}$ & \\
\hline \multirow{3}{*}{ 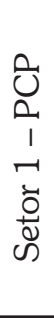 } & $\begin{array}{c}\text { Contratos de } \\
\text { Relacionamento } \\
\text { com Clientes }\end{array}$ & 19,51 & 13,01 & 13,01 & 19,52 & 65,05 \\
\hline & $\begin{array}{c}\text { Contratos de } \\
\text { Franquia } \\
\end{array}$ & 13,11 & 13,11 & 2,18 & 6,55 & 34,95 \\
\hline & $\begin{array}{c}\text { ¿Margem de } \\
\text { Contribuição (\%) }\end{array}$ & 32,61 & 32,61 & 15,19 & 26,06 & 100,00 \\
\hline
\end{tabular}

Fonte: Adaptada de Teixeira (2005)

Tabela 7: Exemplo de Matriz de Margem de Contribuição dos Elementos dos AIIs - Setor 2

\begin{tabular}{|c|c|c|c|c|c|c|}
\hline \multirow[b]{2}{*}{ 息 } & \multirow[b]{2}{*}{ AII Crítico } & \multicolumn{4}{|c|}{ ELEMENTOS DOS AIIs } & \multirow{2}{*}{$\begin{array}{c}\sum \text { Mar- } \\
\text { gem de } \\
\text { Contri- } \\
\text { buição } \\
\text { (\%) }\end{array}$} \\
\hline & & $\begin{array}{l}\text { Recursos } \\
\text { Humanos }\end{array}$ & Processos & $\begin{array}{c}\text { Estrutura } \\
\text { Organiza- } \\
\text { cional }\end{array}$ & $\begin{array}{c}\text { Fatores } \\
\text { Ambien- } \\
\text { tais }\end{array}$ & \\
\hline \multirow{3}{*}{ 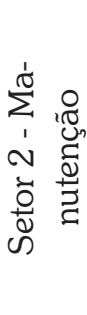 } & $\begin{array}{c}\text { Contratos de } \\
\text { Relacionamento } \\
\text { com Clientes }\end{array}$ & 28,56 & 28,56 & 14,28 & 14,28 & 85,68 \\
\hline & $\begin{array}{c}\text { Contratos de } \\
\text { Franquia }\end{array}$ & 4,09 & 4,09 & 4,09 & 2,05 & 14,32 \\
\hline & $\begin{array}{c}\text { EMargem de } \\
\text { Contribuição (\%) }\end{array}$ & 32,65 & 32,65 & 18,37 & 16,33 & 100,00 \\
\hline
\end{tabular}

Fonte: Adaptada de Teixeira (2005) 
Com os cálculos das margens de contribuição de cada elemento que compõe os Alls considerados críticos à manufatura (por Setor), objetiva-se a busca de informações que possam estabelecer prioridades na aplicação de recursos nos elementos formadores desses AIls. Estabelecendo-se um ordenamento para os Alls críticos e, consequentemente, para seus elementos mais significativos, é possível obter os seguintes resultados para os setores em análise:

Para o Setor 1:

$1^{\circ}$ - AII (Contratos de Relacionamentos com Clientes) - $1^{\circ}$ AII mais crítico, com maior $\mathrm{MC}=65,05 \%$ (ver exemplo em Tabela 6 ), sendo os elementos mais influentes para este AII:

a) Fatores Ambientais - $\mathrm{MC}=19,52 \%$;

b) Recursos Humanos - MC = 19,51\%;

c) Processos - MC = 13,01\%;

d) Estrutura Organizacional - MC $=13,01 \%$;

$2^{\circ}$ - AII (Contratos de Franquia) - $2^{\circ}$ AII mais crítico, com MC $=34,95 \%$ (ver exemplo em Tabela 6), sendo os elementos mais influentes para este AII:

a) Recursos Humanos - MC = 13,11\%;

b) Processos - MC = 13,11;

\section{Para o Setor 2:}

$1^{\circ}$ - AII (Contratos de Relacionamentos com Clientes) - $1^{\circ}$ All mais crítico, com maior $\mathrm{MC}=85,68 \%$ (ver exemplo em Tabela 7 ), sendo os elementos mais influentes para este AII:

a) Recursos Humanos e Processos, ambos com margens de contribuição idênticas de 28,56\%;

$2^{\circ}$ - AII (Contratos de Franquia) - $2^{\circ}$ AII mais crítico, com MC $=14,32 \%$ (ver exemplo em Tabela 7), sendo os elementos mais influentes para este AII:

a) Recursos Humanos, Processos, Estrutura Organizacional, todos com margens de contribuição de 4,09\%. 


\section{Considerações Finais}

Com as proposições descritas, visa-se demonstrar formas de mapeamento da contribuição dos ativos intangíveis, através da determinação de seus valores dentro do contexto organizacional, bem como o desenvolvimento de indicadores organizacionais próprios para estes mesmos ativos.

Com a descrição da metodologia de Teixeira (2005) obtém-se a possibilidade de determinação de quais ativos intangíveis internos sejam considerados críticos ao aumento da eficiência das operações de manufatura, sendo esta a mais ajustada para o desenvolvimento de indicadores que possam evidenciar a contribuição desses ativos, amparada pela utilização da metodologia de Hoss (2003) que define os elementos representativos dos ativos intangíveis representados pelo fator humano, o conjunto de processos, a concepção da estrutura organizacional e os fatores relativos ao ambiente.

Dessa forma, é possível relacionar indicadores desempenho da manufatura aos ativos intangíveis internos organizacionais, determinando graus de criticidade e níveis de importância para cada indicador desenvolvido, projetando a aplicação de recursos financeiros em elementos constitutivos desses mesmos ativos, tendo como objeto final a projeção de um aumento da eficiência operacional no ambiente que constitui a manufatura.

\section{A Contribution for the Development of Performance Indicators of the Organization Intangible Assets}

\section{Abstract}

This paper classifies management models whose original concepts are used and adapted as a way to raise awareness of the importance of internal intangible assets of an organization as effective elements of $\neq$ the economic sustainability of a company. Methodologies considered for this purpose are as follows, the methodology of Teixeira (2005) to determine the manufacture flexibility indicators, the methodology of França (2004) to establish indicators for the organizational intangible assets and the 
methodology and the Hoss (2003) for quantity and quality valuation of intangible assets which are analyzed. An adaption of these methodologies is thus proposed so that they may be customised for the utilization of the knowledge assets. The primary aim of the work presented in this paper, is to be able to assess the real value of the organizational intangible assets and the development of performance indicators for these same assets, in order to obtain better levels of operational efficiency in the management of the industrial context within the a unit manufacture.

Key words: Performance Indicators. Intangible Assets. Manufacture and Organization Objectives.

\section{Referências}

DIAS JUNIOR, C. M.; POSSAMAI, O.; GONÇALVES, R. Using Organizational Intangible Assets for Better Levels of Operational Efficiency. European Ideas for Bussiness - Erima, Biarritz, France, 2007a.

DIAS JUNIOR, C. M.; POSSAMAI, O.; GONÇALVES, R. Systematic for Increase of the Operational Efficiency from the Allocation of Resources in Intangible Assets. Complex Systems Concurrent Engineering: Collaboration, Technology Innovation and Sustainability, Part 13, p. 665-672. São Paulo: Springer, $2007 b$.

FRANÇA, R. B. Avaliação de indicadores de ativos intangíveis. 187f. Tese de Doutorado. Programa de Pós-graduação em Engenharia de Produção e Sistemas, Universidade Federal de Santa Catarina, Brasil, 2004.

HOSS, O. Modelo de avaliação de ativos intangíveis para instituições de ensino superior privado. 159f. Tese de Doutorado. Programa de Pós-graduação em Engenharia de Produção e Sistemas, Universidade Federal de Santa Catarina, Brasil, 2003.

PANDOLFI, Marcos. Sistemas de medição e avaliação de desempenho organizacional: contribuição para gestão de metas globais de performances individuais. 279f. Tese de Doutorado. Escola Politécnica de São Paulo Departamento de Engenharia de Produção, São Paulo, 2005. 
SILVA, C. E. S. Método para avaliação do desempenho do processo de desenvolvimento de produtos. 187f. Tese de Doutorado. Programa de Pósgraduação em Engenharia de Produção e Sistemas, Universidade Federal de Santa Catarina, Brasil, 2001.

TEIXEIRA, R. N. C. Desenvolvimento de um modelo para o planejamento de investimentos em flexibilidade de manufatura em situações de mudanças estratégicas da organização. 295f. Tese de Doutorado. Programa de Pósgraduação em Engenharia de Produção e Sistemas, Universidade Federal de Santa Catarina, Brasil, 2005. 